\title{
AN EMPIRICAL CONNECTION BETWEEN THE ULTRAVIOLET COLOR OF EARLY-TYPE GALAXIES AND THE STELLAR INITIAL MASS FUNCTION
}

\author{
Dennis Zaritsky ${ }^{1}$, Armando Gil de Paz ${ }^{2}$, and Alexandre Y. K. Bouquin ${ }^{2}$ \\ ${ }^{1}$ Steward Observatory, University of Arizona, 933 North Cherry Avenue, Tucson, AZ 85721, USA; dennis.zaritsky@ gmail.com \\ ${ }^{2}$ Departamento de Astrofísica y CC. de la Atmósfera, Facultad de CC. Físicas, Universidad Complutense de Madrid, \\ Avda. de la Complutense s/n, Madrid E-28040, Spain \\ Received 2013 October 8; accepted 2013 October 23; published 2013 December 10
}

\begin{abstract}
Using new UV magnitudes for a sample of early-type galaxies (ETGs) with published stellar mass-to-light ratios, $\Upsilon_{*}$, we find a correlation between UV color and $\Upsilon_{*}$ that is tighter than those previously identified between $\Upsilon_{*}$ and either the central stellar velocity dispersion, metallicity, or alpha enhancement. The sense of the correlation is that galaxies with larger $\Upsilon_{*}$ are bluer in the UV. We conjecture that differences in the lower mass end of the stellar initial mass function (IMF) are related to the nature of the extreme horizontal branch populations that are generally responsible for the UV flux in ETGs. If so, then UV color can be used to identify ETGs with particular IMF properties and to estimate $\Upsilon_{*}$.
\end{abstract}

Key words: galaxies: elliptical and lenticular, cD - galaxies: evolution - galaxies: stellar content - stars: luminosity function, mass function

Online-only material: color figures

\section{INTRODUCTION}

We present and discuss data that are integral to two key unresolved questions regarding the stellar populations of earlytype galaxies (ETGs). Do variations exist among the stellar initial mass functions (IMFs) of ETGs? What is the physical mechanism for producing the stars that give rise to the UV flux in ETGs?

A spate of recent results suggest that the stellar IMF of giant ETGs has more low mass stars for a given total stellar mass than predicted even by the relatively extreme Salpeter mass function $\left(d N / d M \propto M^{-2.35}\right)$. Specifically, van Dokkum \& Conroy (2010), Spiniello et al. (2012), and Ferreras et al. (2013) reach such a conclusion using spectral line indices that are sensitive to the dwarf-to-giant ratio. Independently, Cappellari et al. (2012) and Läsker et al. (2013) find consistent results using dynamical models to measure the total mass. These results are to be contrasted with the extensive evidence in the local neighborhood for an IMF that turns over at sub-solar stellar masses (Bastian et al. 2010).

Although the evidence for variations in the bottom portion of the IMF now extends beyond studies of ETGs, with stellar clusters providing some of the most direct evidence (Strader et al. 2011; Zaritsky et al. 2012, 2013), and direct imaging that resolves sub-solar mass stellar populations promising eventually to settle the matter (Kalirai et al. 2013), the analysis of ETGs is critical in our efforts to understand galaxy evolution.

The second of our two questions traces its origin to the observation of an unexpectedly large UV flux from ETGs (Code \& Welch 1979). Explanations proposed for this "UV-excess" or "UV-upturn" fall into two classes ever since the discovery paper: young stars (Gunn et al. 1981; Rocca-Volmerange \& Guiderdoni 1987) and hot evolved stars. Although in some cases there may be a connection to recent, residual star formation (Yi et al. 2005; Boselli et al. 2005), this explanation does not account for the majority of the UV-upturns (Ferguson et al. 1991; Brown et al. 1997, 2000; Boselli et al. 2005; Han et al. 2007) even though it plays a more prominent role in intermediate and low mass ETGs
(Boselli et al. 2005; Kannappan et al. 2009) and in lenticulars (Salim et al. 2012).

Various scenarios involving evolved stars have been put forward including, post-asymptotic giant branch (AGB) stars (Rose \& Tinsley 1974), hot horizontal branch stars (Ciardullo \& Demarque 1978; Bruzual 1983), and accreting white dwarfs (Greggio \& Renzini 1983). Filling in the details of these populations has proved difficult, but a preliminary consensus is that hot, or extreme, horizontal branch (EHB) stars must be the key contributor to the UV fluxes of ETGs (Han et al. 2007; Lisker \& Han 2008). Although models depend on numerous poorly constrained parameters (mass ratio distribution, tidal atmospheric stripping efficiency), they manage to reproduce several key observational characteristics of the UV-upturn population and have the benefit of relying on a population of objects that are directly observed to exist (Brown et al. 2000; Lisker \& Han 2008; Buzzoni et al. 2012).

The relevance of the UV-upturn extends beyond the nature of EHB stars. For example, models of EHB evolution may inform how the AGB is populated, which will affect how to compute the mass-to-light ratio in bands where the AGB contribution is significant (see Buzzoni \& González-Lópezlira 2008). In general, investigators are focusing on the evolution of binary systems (Han et al. 2007; Lisker \& Han 2008) as a path to the formation of EHB stars. As expected, some of these models do predict a connection between UV excesses and AGB properties (Buzzoni \& González-Lópezlira 2008), thereby implying an effect on calculations of the mass-to-light ratio. Conversely, variations in the IMF could directly affect the UV-upturn population, particularly if binary systems with large mass ratios are an important progenitor class for EHBs. Our two questions are, therefore, intricately connected.

UV-upturn properties vary widely among galaxies (see Code \& Welch 1979; Burstein et al. 1988; Gil de Paz et al. 2007; Bureau et al. 2011). Among the parameters that have been related to the strength of the UV-upturn are (1) stellar population age, which is particularly relevant if some fraction of the UV-bright population is relatively young (Yi et al. 2005; 
Kaviraj et al. 2007; Schawinski et al. 2007), (2) galaxy mass, which is relevant if the galaxy's evolutionary history and total stellar mass are tied to each other (Boselli et al. 2005), (3) stellar density, which is relevant if density-dependent binary processes play a role in forming EHB stars and densities are sufficiently large (see Peacock et al. 2011, for evidence of such a relationship in globular clusters), and (4) metallicity, which is relevant because chemical abundance plays a role in stellar evolution, particularly in late-stage evolution where mass loss is significant. Interestingly, a promising correlation was found between UV properties and metallicity, with more prominent upturns seen in galaxies with greater $\mathrm{Mg}_{2}$ Lick index (Faber 1983; Burstein et al. 1988), an index which broadly tracks metallicity even though it does have some sensitivity to gravity. It is critical to continue the search for other patterns and investigate all such possibilities as a source of insight into the physical mechanisms at work to tie all of this together into a coherent framework.

We examine a set of well-studied galaxies for which lineindex based stellar mass-to-light ratios, $\Upsilon_{*}$, are already available, as are metallicity diagnostics, internal velocity dispersions, and absolute luminosities (from Conroy \& van Dokkum 2012, and references therein). We present new measurements of the farUV (FUV) and near-UV (NUV) fluxes from Galaxy Evolution Explorer (GALEX) data for this set of galaxies. In Section 2 we discuss the data and in Section 3 we describe the findings of our study.

\section{THE DATA AND MEASUREMENTS}

The parent sample for this study is that of nearby ETGs studied by Conroy \& van Dokkum (2012), which overlaps extensively with the sample defined by the SAURON team (Bureau et al. 2011; Jeong et al. 2012). Our addition to the existing data for this sample is the set of homogeneous measurements of the FUV (1350-1750 $\AA$ ) and NUV (1750-2750 $\AA$ ) photometry made possible by the GALEX satellite (Martin et al. 2005; Morrissey et al. 2005).

We analyze ultraviolet imaging data for the 34 ETGs and the bulge of M 31 in the Conroy \& van Dokkum (2012) sample, where we obtained the most recently pipeline-processed (GR6/7 release) GALEX data from the MAST archive maintained by the Space Telescope Science Institute. For all 35 targets we have imaging data of MIS-depth (one GALEX orbit or more; mainly from the NGS, GI, MIS, and DIS surveys) in the GALEX NUV band and for 32 of them, all except NGC 3414, NGC 4382, and NGC 4564, we also have similarly deep FUV data. The first two of these three galaxies are excluded from our analysis because only shallow ( $\leqslant 100$ s-long exposures) FUV data from the All-sky Imaging Survey exist. In the case of NGC 4564, the FUV total exposure time was $504 \mathrm{~s}$, not quite as long as for the rest of the FUV imaging data but enough for the purpose of this study. We exclude the bulge of M 31 on the grounds that it is significantly different in nature than the other systems.

We follow the procedure described by Gil de Paz et al. (2007) and Lee et al. (2011). In summary, the analysis steps are (1) sky-background subtraction, using elliptical annuli centered on the galaxy that match the ellipticity and position angle (P.A.) of the galaxy and have major axes significantly larger than the isophotal diameter, D25, in all cases (the same region is used for each of the two UV bands), (2) interactive masking of foreground stars and background galaxies following an automated detection of all red (FUV-NUV $>1)$ point sources as potential contaminants, (3) surface photometry within elliptical
Table 1

Galaxy Sample and Properties ${ }^{\mathrm{a}}$

\begin{tabular}{|c|c|c|c|c|}
\hline Name & $m_{\mathrm{FUV}}$ & $m_{\mathrm{NUV}}$ & $\begin{array}{c}\text { Distance } \\
(\mathrm{Mpc})\end{array}$ & Class \\
\hline NGC 474 & $18.32 \pm 0.10$ & $15.93 \pm 0.06$ & 32.0 & B \\
\hline NGC 524 & $17.76 \pm 0.04$ & $15.82 \pm 0.04$ & 23.3 & $\mathrm{R}$ \\
\hline NGC 821 & $18.43 \pm 0.14$ & $16.23 \pm 0.09$ & 23.4 & $\mathrm{~N}$ \\
\hline NGC 1023 & $16.54 \pm 0.08$ & $14.86 \pm 0.06$ & 11.1 & $\mathrm{~N}$ \\
\hline NGC 2549 & $18.38 \pm 0.05$ & $16.47 \pm 0.03$ & 12.3 & $\mathrm{~N}$ \\
\hline NGC 2685 & $15.53 \pm 0.07$ & $15.07 \pm 0.02$ & 15.0 & B \\
\hline NGC 2695 & $18.44 \pm 0.05$ & $17.07 \pm 0.06$ & 31.5 & $\mathrm{~N}$ \\
\hline NGC 2699 & $19.42 \pm 0.04$ & $17.72 \pm 0.04$ & 26.2 & $\mathrm{~N}$ \\
\hline NGC 2768 & $17.05 \pm 0.06$ & $15.22 \pm 0.04$ & 21.8 & $\mathrm{~N}$ \\
\hline NGC 2974 & $17.37 \pm 0.56$ & $15.79 \pm 0.26$ & 20.9 & $\mathrm{~N}$ \\
\hline NGC 3377 & $17.18 \pm 0.27$ & $15.08 \pm 0.02$ & 10.9 & $\mathrm{~N}$ \\
\hline NGC 3379 & $16.35 \pm 0.01$ & $14.82 \pm 0.02$ & 10.3 & $\mathrm{~N}$ \\
\hline NGC 3384 & $17.34 \pm 0.05$ & $15.27 \pm 0.03$ & 11.3 & $\mathrm{~N}$ \\
\hline NGC 3608 & $17.70 \pm 0.03$ & $16.20 \pm 0.04$ & 22.3 & $\mathrm{~N}$ \\
\hline NGC 4262 & $17.06 \pm 0.04$ & $16.01 \pm 0.04$ & 15.4 & B \\
\hline NGC 4270 & $19.32 \pm 0.10$ & $17.46 \pm 0.02$ & 33.1 & $\mathrm{~N}$ \\
\hline NGC 4278 & $16.32 \pm 0.04$ & $15.32 \pm 0.03$ & 15.6 & $\mathrm{~N}$ \\
\hline NGC 4458 & $19.25 \pm 0.10$ & $17.18 \pm 0.03$ & 16.4 & $\mathrm{~N}$ \\
\hline NGC 4459 & $17.22 \pm 0.04$ & $15.54 \pm 0.04$ & 16.1 & $\mathrm{~N}$ \\
\hline NGC 4473 & $17.19 \pm 0.04$ & $15.54 \pm 0.03$ & 15.3 & $\mathrm{~N}$ \\
\hline NGC 4486 & $14.64 \pm 0.01$ & $13.85 \pm 0.02$ & 17.2 & $\mathrm{~N}$ \\
\hline NGC 4546 & $17.65 \pm 0.04$ & $15.78 \pm 0.02$ & 13.7 & $\mathrm{~N}$ \\
\hline NGC 4552 & $15.97 \pm 0.03$ & $15.02 \pm 0.01$ & 15.8 & $\mathrm{~N}$ \\
\hline NGC 4564 & $17.72 \pm 0.03$ & $16.38 \pm 0.07$ & 15.8 & $\mathrm{~N}$ \\
\hline NGC 4570 & $17.65 \pm 0.02$ & $16.13 \pm 0.02$ & 17.1 & $\mathrm{~N}$ \\
\hline NGC 4621 & $16.61 \pm 0.05$ & $15.24 \pm 0.08$ & 14.9 & $\mathrm{R}$ \\
\hline NGC 4660 & $17.81 \pm 0.03$ & $16.08 \pm 0.04$ & 15.0 & $\mathrm{~B}$ \\
\hline NGC 5308 & $18.51 \pm 0.03$ & $16.99 \pm 0.02$ & 34.1 & $\mathrm{~N}$ \\
\hline NGC 5813 & $17.14 \pm 0.03$ & $15.58 \pm 0.04$ & 31.3 & $\mathrm{~N}$ \\
\hline NGC 5838 & $17.77 \pm 0.06$ & $16.20 \pm 0.02$ & 19.8 & $\mathrm{~N}$ \\
\hline NGC 5845 & $19.15 \pm 0.05$ & $17.87 \pm 0.03$ & 25.2 & $\mathrm{~N}$ \\
\hline NGC 5846 & $16.52 \pm 0.04$ & $15.48 \pm 0.04$ & 24.2 & $\mathrm{R}$ \\
\hline
\end{tabular}

Note. ${ }^{a}$ The quoted uncertainties do not include the zero point uncertainties, but a systematic zero point error would affect all measurements equally and so do not affect the conclusions presented here. Class refers to our UV-optical color based classification as normal (N), blue outlier (B), or red outlier (R).

annuli with fixed center, ellipticity and P.A. (those of the D25 ellipse), and (4) calculation of the growth curve in both UV bands and the derivation of the corresponding asymptotic magnitudes. We use the FUV and NUV asymptotic magnitudes as the best measure of the total UV emission of our galaxies (Table 1). Distances are adopted from the on-line database NED.

\section{RESULTS}

We present the UV properties of the sample in Figure 1 and Table 1. The top panel of the figure shows the relation between $M_{I}$ and $M_{\mathrm{NUV}}$, confirming that the UV flux is generally related to the properties of the galaxy as a whole and is not the product of UV "froth" resulting from random low levels of recent star formation. We highlight the most striking outliers above the relationship (UV-bright) using blue triangles. In the bottom panel we explore the nature of these outliers in color-magnitude space. These outliers are clearly UV-bright and UV-blue and may be examples of galaxies where recent star formation is affecting the total photometry. We also note three galaxies that are redder than the red sequence in this panel, which are highlighted using red squares. These objects might represent cases where reddening is somewhat more prevalent, or alternatively galaxies with different mean stellar populations 


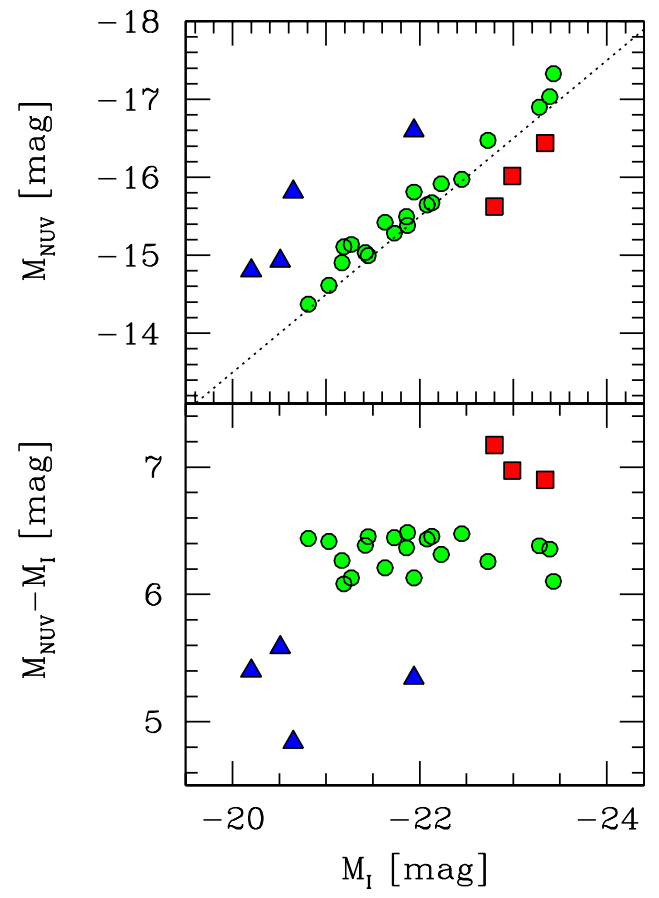

Figure 1. NUV properties of sample galaxies. Galaxies noted with blue circles or red squares are outliers described in text. Upper panel shows that for the bulk of the sample, the NUV luminosities tightly follow the I-band luminosities, demonstrating that the UV flux is not due to "froth" from random low levels of residual star formation. The bottom panel illustrates how we selected our red and blue outliers.

(A color version of this figure is available in the online journal.)

due to a factor such as mean metallicity. The latter supposition is not supported by the available data.

The surprising result comes in the far right panel of Figure 2, where we show the relationship between UV color (FUV NUV) and $\Upsilon_{*}$. The relationship between FUV - NUV and $\Upsilon_{*}$ is incredibly tight (Spearman $R=0.78$, excluding one outlier-see below), tighter even than that between $\Upsilon_{*}$ and the velocity dispersion, $\sigma_{V}$, found by Conroy \& van Dokkum (2012) and used to motivate the finding of a mass dependent IMF (see Table 2 for a summary of the Spearman rank correlation coefficients in the lower half of the matrix and the probability that correlation coefficients that are at least as large as those measured arise by chance in the upper half). The one notable exception to the tight relationship between FUV - NUV and $\Upsilon_{*}$ is NGC 2685, which is known as the "Spindle" galaxy for its odd morphology (Sandage 1961) and is modeled as a multiplyringed polar-ring galaxy (Józsa et al. 2009). We have removed this one object from all of the correlation calculations in Table 2, but include it in all of the figures.

The galaxies we identified as the blue outliers are NGC 474, 2685, 4262, 4660. These are all well-known S0 galaxies, three of which show clear interaction signatures. We discussed NGC 2685 above and NGC 4262 has an extended UV ring (Bettoni et al. 2010). NGC 474 is classified as peculiar on the basis of its shells (Buta et al. 2010) and identified as having residual star formation by Jeong et al. (2012). The other three of our blue outliers are not among the galaxies classified by Jeong et al. (2012), but three other galaxies from our sample (NGC 1023, NGC 2974, and NGC 4459) are identified as having residual star formation, although these are manifestly not large outliers in Figure 1. Of the four blue outliers that we identified, NGC 4660, is the most normal in appearance and its large disk component was only discovered through its kinematics (Rix \& White 1990). We suspect that the unusual UV properties of these four are related to recent, and perhaps unusually strong, interactions rather than the $\mathrm{S} 0$ nature of the galaxies themselves because the three red outliers (NGC 524, NGC 4621, and NGC 5846) are also S0 galaxies and lack clear interaction signatures. Interestingly, "blue" S0's are typically difficult to find in the types of environment where S0's are thought to be forming (for example, see Just et al. 2011) and may represent a stage following the E+A or post-starburst phase (Yang et al. 2008). Nevertheless, with the exception of NGC 2685, which is quite an unusual galaxy, the relationship between the UV-upturn color and $\Upsilon_{*}$ holds.

Of the other correlations explored in Table 2 and Figure 2, the only the ones, with one exception, that are statistically significant are those with $\sigma_{V}$ and $\langle\mathrm{Mg} / \mathrm{Fe}\rangle$, but they are quantitatively weaker than that between FUV - NUV and $\Upsilon_{*}$. The one exception is the correlation between $\langle\mathrm{Fe} / \mathrm{H}\rangle$ and $\langle\mathrm{Mg} / \mathrm{Fe}\rangle$, which is trivial because the two quantities both depend on Fe. Even so, it is weaker than that between FUV - NUV and $\Upsilon_{*}$.

We are concerned that the extremely strong correlation between FUV - NUV and $\Upsilon_{*}$ results from a systematic error in
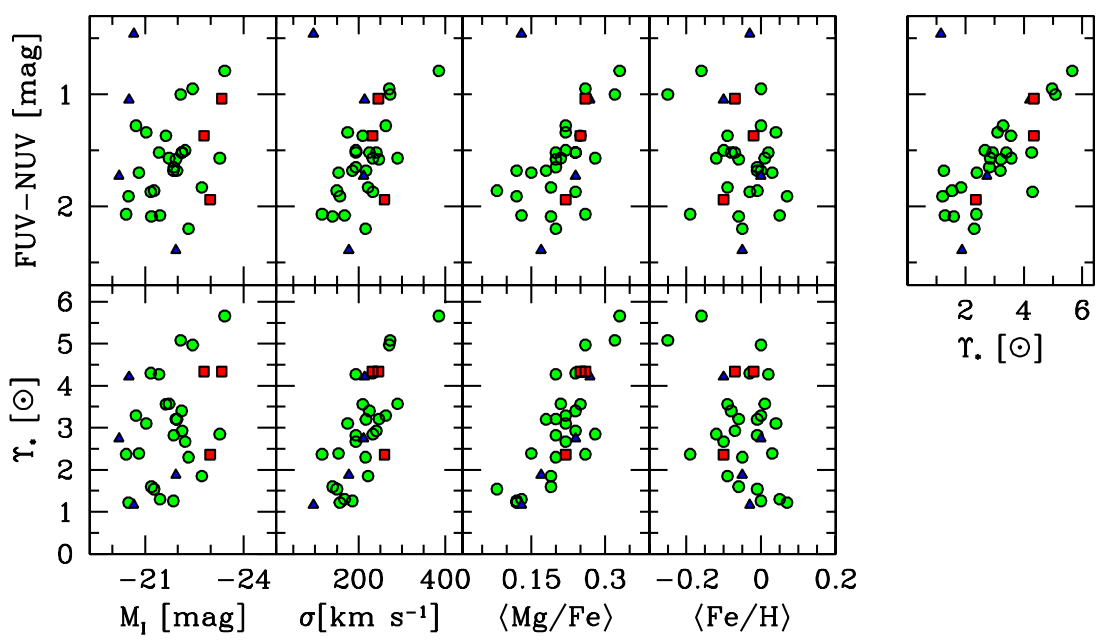

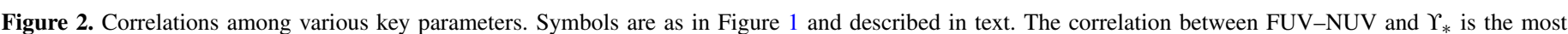
statistically significant among this set. With one exception (NGC 2685), the color outliers follow the general patterns.

(A color version of this figure is available in the online journal.) 
Table 2

Rank Correlation Coefficients and Probabilities They Arise Randomly

\begin{tabular}{lcccccc}
\hline \hline & FUV - NUV & $M / L_{I}$ & $L_{I}$ & $\sigma$ & $\langle\mathrm{Mg} / \mathrm{Fe}\rangle$ & $\langle\mathrm{Fe} / \mathrm{H}\rangle$ \\
\hline FUV - NUV & $\ldots$ & $\mathbf{6 . 3} \times \mathbf{1 0}^{-\mathbf{8}}$ & 0.49 & $\mathbf{2 . 0} \times \mathbf{1 0}^{-\mathbf{3}}$ & $\mathbf{3 . 1} \times \mathbf{1 0}^{-\mathbf{5}}$ & 0.49 \\
$M / L_{I}$ & -0.78 & $\ldots$ & 0.23 & $\mathbf{4 . 4} \times \mathbf{1 0}^{-\mathbf{5}}$ & $\mathbf{2 . 0} \times \mathbf{1 0}^{-\mathbf{6}}$ & 0.25 \\
$L_{I}$ & 0.12 & -0.21 & $\ldots$ & $\mathbf{8 . 9} \times \mathbf{1 0}^{-\mathbf{3}}$ & 0.31 & 0.05 \\
$\sigma$ & -0.51 & 0.64 & -0.44 & $\ldots$ & $\mathbf{3 . 1} \times \mathbf{1 0}^{-\mathbf{4}}$ & 0.03 \\
$\langle\mathrm{Mg} / \mathrm{Fe}\rangle$ & -0.65 & 0.72 & -0.18 & 0.58 & $\ldots$ & $\mathbf{4 . 3}^{-3} \mathbf{1 0}^{-\mathbf{5}}$ \\
$\langle\mathrm{Fe} / \mathrm{H}\rangle$ & 0.12 & -0.20 & 0.33 & -0.38 & -0.64 & $\ldots$ \\
\hline
\end{tabular}

Note. ${ }^{\text {a }}$ Rank correlation coefficients given in bottom half of matrix, probabilities in top half. Significant correlation highlighted in bold.

the derivation of $\Upsilon_{*}$ caused either directly or indirectly by the population responsible for the UV-upturn. A direct influence is unlikely as any black body responsible for the UV flux would provide negligible flux in the $I$-band where the IMF sensitive lines lie. More plausible is that the presence of UV emitting stars is related to a "distortion" of the AGB or red giant branch (RGB) population, either affecting the line indices or lowering the $I$-band flux below expectations. The former, an effect on the line indices, cannot be responsible because the variation in $\Upsilon_{*}$ is also inferred from kinematic analyses (Cappellari et al. 2012; Läsker et al. 2013). If, however, a lower-than-expected I-band flux is the rule in galaxies with large UV-upturns, then both the line-index and kinematic method will yield higher $\Upsilon_{*}$ for those galaxies. In such a scenario, the measurement of $\Upsilon_{*}$ would not be in error, but the inference that variations in $\Upsilon_{*}$ are related to a change in the IMF would be.

We now discuss two scenarios that attempt to explain the empirical findings with and without IMF variations. First, consider the situation where the UV upturn stars come directly from the population of $\mathrm{AGB} / \mathrm{RGB}$ stars, which is to say that some process converts $\mathrm{AGB} / \mathrm{RGB}$ stars into UV-upturn stars, and this phenomenon is not included in current spectral synthesis models. In such a scenario, proportionally more UV upturn stars in a certain galaxy means proportionally fewer AGB/RGB stars, and therefore a lower $I-$ band luminosity. A relationship in this sense is found in at least one model of EHB evolution (Buzzoni \& González-Lópezlira 2008), although it is unclear whether that particular model will work quantitatively for the UV upturn- $\Upsilon_{*}$ relationship we observe. Other possibilities, for example one in which chemical abundance plays a role through winds and mass transfer rates, would also help explain other observables such that of larger UV-upturn populations in galaxies with higher $\mathrm{Mg}_{2}$. Second, consider the situation where low mass stars in multiple star systems play a key role in transforming more massive evolved stars into the EHB stars of the UV-upturn population. In such a scenario, having more low mass stars in a bottom-heavy stellar population will lead to a larger UV-upturn population in the galaxies with higher $\Upsilon_{*}$-again in qualitative agreement with the observations. We cannot, with the data discussed so far, distinguish between these two possibilities.

Photometric data in other passbands might help resolve the situation. In a scenario where the I-band luminosity is different than the model expectations-whether the origin of that discrepancy is physical or observational-we might expect to see significant deviations in colors involving the $I$-band. We obtain $H$-band data for much of the sample from Two Micron All Sky Survey (collated from the NED database) and find no significant correlation between $I$-band and $I-H$. We conclude that variations in $I$-band luminosities alone are an unlikely origin for our findings.
Given (1) that the $\Upsilon_{*}$ correlation with $\sigma_{V}$ is observed when $\Upsilon_{*}$ is either measured spectroscopically (Conroy \& van Dokkum 2012) or kinematically (Cappellari et al. 2012), (2) the lack of any signature of odd behavior in the $I$-band luminosities as reflected in $I-H,(3)$ the lack of a dependence of the $\Upsilon_{*}$ of old $(\log ($ age $)>9.8)$ Local Group stellar clusters with $\langle\mathrm{Mg} / \mathrm{Fe}\rangle$ (Cameron 2009; Colucci et al. 2012; Zaritsky et al. 2012, 2013), and (4) that the strongest correlation we find is between $\Upsilon_{*}$ and UV color, we conclude that the variation in $\Upsilon_{*}$ is real, and that it is directly correlated to UV color. We suggest that IMF variations are responsible for the differences in the EHB populations of ETGs. Nevertheless, we need to remain cognizant of the possibility that other physical effects beside IMF variations are responsible for the various correlations (see Conroy \& van Dokkum 2012). The possibility that metallicity is somehow responsible for both the IMF and UV-upturn variations remains a real possibility.

\section{CONCLUSION}

Using GALEX data we have identified a strong correlation between UV color and the stellar mass to light ratio, $\Upsilon_{*}$ in ETGs. This correlation is stronger, within the same sample, than those previously identified between $\Upsilon_{*}$ and either $\sigma_{V}$, metallicity, or alpha enhancement.

We are faced with that task of explaining an interrelated set of significant correlations between $\sigma_{V},\langle\mathrm{Mg} / \mathrm{Fe}\rangle, \Upsilon_{*}$, and UV color. It is inherently difficult to draw conclusions from a quantitative comparison among statistically significant correlations because the measured strength of the correlations depends on the magnitude of the observational uncertainties. We hypothesize that the variations in the IMF identified in previous investigations are driving the correlation between $\Upsilon_{*}$ and UV color, but the role of metallicity in both is still unknown. As is usually the case with empirical correlations, understanding their origin and determining whether a correlation implies causality is a more difficult task than the discovery of the correlation.

A final intriguing possibility is the use of the UV color as a tracer of IMF variations in galaxies without recent star formation, at least as a selection criteria with which to identify galaxies for further study.

D.Z. acknowledges financial support from NASA ADAP NNX12AE27G and NSF AST-1311326, and NYU CCPP for its hospitality during long-term visits. The authors acknowledge the support from the FP7 Marie Curie Actions of the European Commission, via the Initial Training Network DAGAL under REA grant agreement PITN-GA-2011-289313. This research has made use of the NASA/IPAC Extragalactic Database 
(NED), which is operated by the Jet Propulsion Laboratory, California Institute of Technology, under contract with NASA.

\section{REFERENCES}

Bastian, N., Covey, K. R., \& Meyer, M. R. 2010, ARA\&A, 48, 339

Bettoni, D., Buson, L. M., \& Galleta, G. 2010, A\&A, 519, 72

Boselli, A., Cortese, L., Deharveng, J. M., et al. 2005, ApJL, 629, L29

Brown, T. M., Bowers, C. W., Kimble, R. A., Sweigart, A. V., \& Ferguson, H. C. 2000, ApJ, 532, 308

Brown, T. M., Ferguson, H. C., Davidsen, A. F., \& Dorman, B. 1997, ApJ, 482, 685

Bruzual, G. 1983, ApJ, 273, 105

Bureau, M., Jeong, H., Yi, S. K., et al. 2011, MNRAS, 414, 1887

Burstein, D., Bertola, F., Buson, L. M., Faber, S. M., \& Lauer, T. R. 1988, ApJ, 328,440

Buta, R. J., Sheth, K., Regan, M., et al. 2010, ApJS, 190, 147

Buzzoni, A., Bertone, E., Carraro, G., \& Buson, L. 2012, ApJ, 749, 35

Buzzoni, A., \& González-Lópezlira, R. A. 2008, ApJ, 666, 1007

Cameron, S. A. 2009, PhD thesis, Univ. Michigan

Cappellari, M., McDermid, R. M., Alatalo, K., et al. 2012, Natur, 484, 485

Ciardullo, R. B., \& Demarque, P. 1978, in IAU Symp. 80, The HR Diagram, ed. A. G. D. Philip \& D. S. Haynes (Dordrecht: Reidel), 345

Code, A. D., \& Welch, G. A. 1979, ApJ, 228, 95

Colucci, J. E., Bernstein, R. A., Cameron, S. A., \& McWilliam, A. 2012, ApJ, 746,29

Conroy, C., \& van Dokkum, P. G. 2012, ApJ, 760, 71

Faber, S. M. 1983, HiA, 6, 165

Ferguson, H. C., Davidsen, A. F., Kriss, G. A., et al. 1991, ApJ, 382, 69

Ferreras, I., La Barbera, F., de la Rosa, I. G., et al. 2013, MNRAS, 429, 15L

Gil de Paz, A., Boissier, S., Madore, B. F., et al. 2007, ApJS, 173, 185

Greggio, L., \& Renzini, A. 1983, A\&A, 118, 217

Gunn, J. E., Stryker, L. L., \& Tinsley, B. M. 1981, ApJ, 249, 48
Han, Z., Podsiadlowski, Ph., \& Lynas-Gray, A. E. 2007, MNRAS, 380, 1098 Jeong, H., Yi, S. K., Bureau, M., et al. 2012, MNRAS, 423, 1921

Józsa, G. I. G., Osterloo, T. A., Morganti, R., Klein, U., \& Erben, T. 2009, A\&A, 494, 489

Just, D., Zaritsky, D., Tran, K.-V. H., et al. 2011, ApJ, 740, 54

Kalirai, J. S., Anderson, J., Dotter, A., et al. 2013, ApJ, 763, 110

Kannappan, S. J., Guie, J. M., \& Baker, A. J. 2009, AJ, 138, 579

Kaviraj, S., Schawinski, K., Devriendt, J. E. G., et al. 2007, ApJS, 173, 619

Läsker, R., van den Bosch, R. C. E., van de Ven, G., et al. 2013, MNRAS, 434, L31

Lee, J. C., Gil de Paz, A., Kennicutt, R. C., Jr., et al. 2011, ApJS, 192, 6

Lisker, T., \& Han, Z. 2008, ApJ, 680, 1042

Martin, D. C., Fanson, J., Schiminovich, D., et al. 2005, ApJL, 619, L1

Morrissey, P., Schiminovich, D., Barlow, T. A., et al. 2005, ApJL, 619, L7

Peacock, M. B., Maccarone, T. J., Dieball, A., \& Knigge, C. 2011, MNRAS, 411, 487

Rix, H.-W., \& White, S. D. M. 1990, ApJ, 362, 52

Rocca-Volmerange, B., \& Guiderdoni, B. 1987, A\&A, 175, 15

Rose, W. B., \& Tinsley, B. M. 1974, ApJ, 190, 243

Salim, S., Fang, J. J., Rich, R. M., Faber, S. M., \& Thilker, D. A. 2012, ApJ, 755,105

Sandage, A. 1961, The Hubble Atlas of Galaxies (Washington, DC: Carnegie Institute of Washington)

Schawinski, K., Kaviraj, S., Khochfar, S., et al. 2007, ApJS, 173, 512

Spiniello, C., Trager, S. C., Koopmans, L. V. E., \& Chen, Y. P. 2012, ApJ, 753,32

Strader, J., Caldwell, N., \& Seth, A. C. 2011, AJ, 142, 8

van Dokkum, P. G., \& Conroy, C. 2010, Natur, 468, 940

Yang, Y., Zabludoff, A. I., Zaritsky, D., \& Mihos, J. C. 2008, ApJ, 688, 945

Yi, S., Yoon, S.-J., Kaviraj, S., et al. 2005, ApJL, 619, L111

Zaritsky, D., Colucci, J. E., Pessev, P. M., Bernstein, R. A., \& Chandar, R. 2012, ApJ, 761, 93

Zaritsky, D., Colucci, J. E., Pessev, P. M., Bernstein, R. A., \& Chandar, R. 2013, ApJ, 770, 121 